\title{
On the Primal and Dual Forms of the Stewart Platform Pure Condition
}

\author{
Júlia Borràs and Federico Thomas, Member, IEEE
}

\begin{abstract}
The algebraic characterization of the singularities of a Stewart platform is usually presented as a $6 \times 6$ determinant, whose rows correspond to the line coordinates of its legs, equated to zero. This expression can be rewritten in a more amenable way, known as the pure condition, as sums and products of $4 \times 4$ determinants whose rows correspond to the point coordinates of the legs attachments. Researchers usually rely on one of these two expressions to find the geometric conditions associated with the singularities of a particular Stewart platform. Although both are equivalent, it is advantageous to use either line or point coordinates depending on the platform topology. In this context, an equivalent expression involving only plane coordinates, a dual expression to that using point coordinates, seems to be missing. This paper is devoted to its derivation and to show how its use is advantageous in many practical cases mainly because of its surprising simplicity: it only involves the addition of $4 \times 4$ determinants whose rows are plane coordinates defined by sets of three attachments.
\end{abstract}

Index Terms-Stewart platform, singularities, pure condition, Grassman-Cayley algebra.

\section{INTRODUCTION}

A Stewart platform is a type of parallel robot that incorporates six prismatic actuators, or legs, all of them connected simultaneously to a fixed base and a moving platform through spherical joints, or attachments. It triggered the research on parallel manipulators and continues to be the center of many researches because, despite its simple geometry, its analysis translates into challenging mathematical problems [1]. One of these problems is to determine the configurations in which the moving platform becomes uncontrollable, that is, the singularities. Very large joint forces can occur in the neighborhood of a singularity that may even lead to the breakdown of the robot. Although singularities are usually avoided for obvious reasons, being close to them may be useful in some cases. Indeed, as pointed out in [2], the large amplification factors between the moving platform motion and the actuated joint motion arising in a singularity may be interesting for fine-positioning devices with a very small workspace or for improving the sensitivity along some measurement directions when a platform is used as a force sensor [3]. Thus, having the geometric conditions that lead to a singularity greatly simplifies the design phase of a Stewart platform no matter if we want to avoid them or to take advantage of them. It is also worth mentioning that one of the associated problems with the characterization of the singularities of a Stewart platform is the definition of a performance index representing the closeness to the singularities. Unfortunately, no mathematical sound metric

The authors are with the Institut de Robòtica i Informàtica Industrial (CSIC-UPC), Llorens Artigas 4-6, 08028 Barcelona, Spain. \{jborras, fthomas\}@iri.upc.edu measuring this closeness exists [4]. Hence, a certain level of arbitrariness must be accepted in its definition which is alleviated if we support it on a geometric meaningful interpretation. This paper is about the problem of using the best formulation for each Stewart platform instance that provides us with the most simple and straightforward geometric interpretation of its singularities.

Different Stewart platform topologies are obtained by coalescing, either on the base or the platform, some of the attachments [5]. Then, a generic Stewart platform is usually referred to as a 6-6 Stewart platform while, on the other side of the spectrum, a 3-3 platform refers to any of the three possible topologies in which a Stewart platform has three attachments both in the base and in the platform. Although coalescing attachments leads to multiple spherical joints whose implementation is difficult [6], studying the singularities for all these possible topologies is of great practical relevance because the singularities of 6-6 platforms with some particular arrangements of attachments [7], and the singularities of some 3-legged robots can be reduced to the study of the singularities of some of these topologies [8], [9].

From the algebraic point of view, the singularities of a Stewart platform arise when its $6 \times 6$ inverse Jacobian $\mathbf{J}$ (the matrix that maps the twist of the platform into the velocities of the actuators) is singular, that is, when its determinant $\operatorname{det}(\mathbf{J})$ is equal to 0. Unfortunately, computing this determinant is difficult even with symbolic computation tools [10], [11]. The advantage of this approach is that once the expression of the determinant is obtained, the locus of singular configurations can be plotted in the workspace. However, the determinant itself is usually a large expression, even for simple topologies, that does not provide insight into the geometric conditions associated with the singularities. Nevertheless, a better understanding of these conditions can be straightforwardly derived from this formulation by using Line Geometry [12]. Since the rows of $\mathbf{J}$ are the Plücker vectors of the lines supporting the robot legs, a singularity of $\mathbf{J}$ implies a linear dependence between these vectors [13], [14], [15], a situation that only occurs if the lines associated with the vectors satisfy particular geometric constraints [16]. The singularity analysis is thus reduced to determining conditions on the pose parameters for which these constraints are satisfied, giving geometric information on the singularity variety [17].

Recently, the use of Grassman-Cayley algebra to study the singularities of Stewart platforms has received a lot of attention [18], [19], [8]. Using this algebra, the determinant of the Jacobian equated to zero can be rewritten as an expression known as the pure condition [20]. It is formed by 24 adding terms of three multiplying brackets each. Each bracket is 
a $4 \times 4$ determinant whose rows correspond to the homogeneous coordinates of an attachment. Thus, each bracket can be interpreted as the oriented volume of the tetrahedron formed by four attachments. The pure condition is unique up to syzygies, a term borrowed from the literature on classic invariant theory, which refer to the three-term GrassmannPlücker relations (see [21] for a gentle introduction to these relations). This means that there are different equivalent pure conditions that vary in their number of terms which somewhat complicates things. For those topologies for which any of these equivalent expressions simplifies to a single term, a straightforward geometric interpretation of the singularities is obtained as degeneracies of three tetrahedra whose vertices coincide with attachments. When such simplification cannot be achieved, the geometric interpretation becomes much more complex. The octahedral topology (one of the three possible 3-3 topologies) was analyzed in [18], where it was shown that its pure condition cannot be simplified to a single term, but a further analysis still using Grassmann-Cayley algebra permitted to characterize its singularity condition as the intersection of four planes. More recently, a similar result was obtained for six more topologies [22]. In all these cases the singularities could also be interpreted as degeneracies of tetrahedra with the difference that their vertices are no longer defined by attachments. Instead, the faces of these tetrahedra are defined by sets of three attachments. Thus, some sort of duality seems to surface from these results. This paper deepens on this duality and, as a result, derives a new singularity condition for the generic 6-6 Stewart platform in terms of the latter kind of tetrahedra.

Certainly there have been other approaches besides the two aforementioned ones to obtain the geometric conditions associated with the singularities. For example, the one presented in [23] is based on a convenient expansion of the determinant of the Jacobian matrix into $3 \times 3$ minors. The obtained singularity condition has 20 terms, each one being the product of two $3 \times 3$ determinants, which can be rewritten as triple products. The resulting expression depends on two types of vectors, one representing director vectors of the leg lines and the other moments of these lines. Such expression is used to obtain the analytical expression of the singularity polynomial of the general Stewart platform and to show one topology for which this expression simplifies to a single term.

This paper is organized as follows. First, Section II briefly reviews some basic concepts on the duality of points and planes and introduces the definitions that will be used throughout this paper. Section III briefly reviews the primal form of the pure condition. Section IV introduces the concept of dual form of the pure condition and presents it for the generic 66 Stewart platform. Section V discuses the pros and cons of using the pure condition either in its primal or dual form to obtain the geometric interpretation of the singularities of a given topology. Section VI presents two examples. Finally, Section VII summarizes the main results and give prospects for further work. An important effort has been done to make the presentation self-contained and understandable without any previous knowledge on Grassmann-Caley algebra.

\section{NOT(AT)IONS}

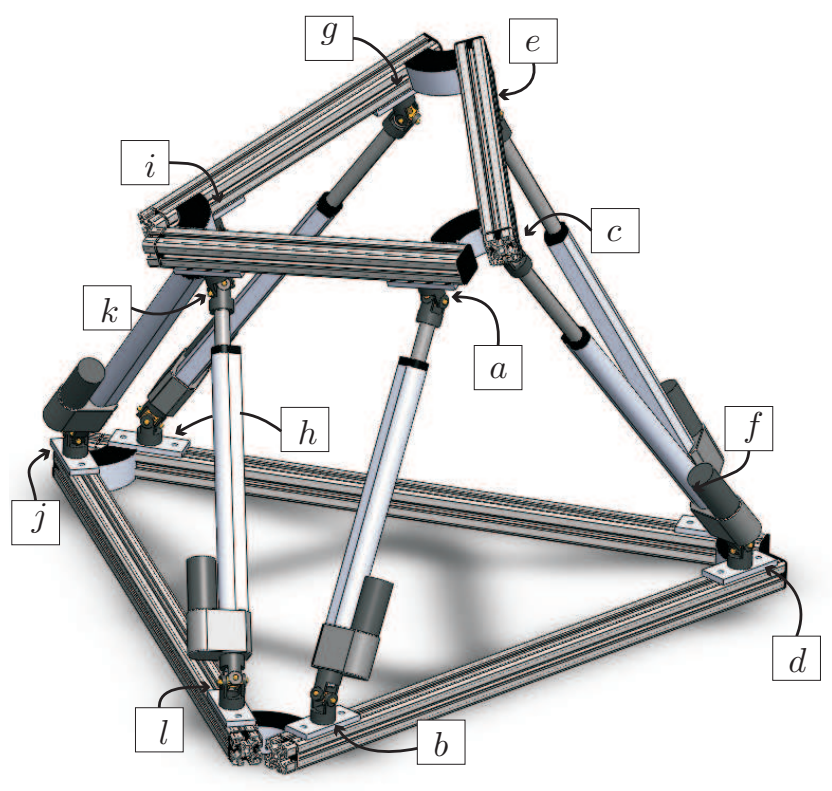

Fig. 1. A 6-6 Stewart platform and adopted labelling for its leg attachments.

The location of the twelve center points of the leg attachments of a 6-6 Stewart platform will be represented by $a, b, c$, $\ldots, l$, as shown in Fig. 1. Non-generic Stewart platforms are obtained by making some leg attachments coplanar, aligned, or simply coincident. In what follows, our analysis will only consider coincidences which can be extended to consider colinearities and coplanarities as described in [24].

To lighten the notation, lowercase Latin letters will represent both points and their position vectors in homogeneous coordinates in a given global reference frame, and lowercase Greek letters, scalars.

As already mentioned in the introduction, the singularities of a Stewart platform correspond to those configurations in which the determinant of the Plücker coordinates of its leg lines is zero, that is, $\operatorname{det}(\mathbf{J})=0$. Now, without loss of generality, let us suppose that $a=\alpha_{1} p+\alpha_{2} q$. That is, the first attachment is known to lie on the line defined by $p$ and $q$. Then, due to the multilinearity of the Jacobian determinant, $\operatorname{det}(\mathbf{J})=$ $\alpha_{1} \operatorname{det}\left(\left.\mathbf{J}\right|_{a=p}\right)+\alpha_{2} \operatorname{det}\left(\left.\mathbf{J}\right|_{a=q}\right)$. This property will be useful later.

Since points will be represented by their homogeneous coordinates, $p=\left(p_{x}, p_{y}, p_{z}, p_{w}\right)^{T}$, they are considered to belong to the projective space $\mathbb{P}^{3}$. Thus, a point in $\mathbb{P}^{3}$ corresponds to a vector in the standard 4-dimensional vector space V. In $\mathbb{P}^{3}$, duality refers to geometric transformations that replace points by planes and planes by points while preserving incidence properties between the transformed objects [25]. The existence of such transformations leads to a general principle, the duality principle, which states that any theorem about incidences between points and planes may be transformed into another theorem about planes and points, by the substitution of the appropriate words [26].

The duality between points and planes is defined by the correspondence $c_{x} x+c_{y} y+c_{z} z+c_{w} w=0 \leftrightarrow\left(c_{x}, c_{y}, c_{z}, c_{w}\right)$. 
That is, the dual point to the plane with implicit equation $c_{x} x+c_{y} y+c_{z} z+c_{w} w=0$ is $c=\left(c_{x}, c_{y}, c_{z}, c_{w}\right)^{T}$. Thus, a plane can be seen as a point and viceversa.

Definition 1 (Cross-product of three vectors) The crossproduct of three vectors $a, b$ and $c$ is defined as:

$$
\lfloor a b c\rfloor=\left|\begin{array}{cccc}
x & y & z & w \\
a_{x} & a_{y} & a_{z} & a_{w} \\
b_{x} & b_{y} & b_{z} & b_{w} \\
c_{x} & c_{y} & c_{z} & c_{w}
\end{array}\right|
$$

where $x=(1,0,0,0)^{T}, y=(0,1,0,0)^{T}, z=(0,0,1,0)^{T}$, and $w=(0,0,0,1)^{T}[27]$.

According to this definition, the plane defined by the three points $a, b$, and $c$ is given by the dual to the point $\lfloor a b c\rfloor$. Then, we also have by duality that the intersection of the dual planes to the points $a, b$, and $c$ is given by $\lfloor a b c\rfloor$ [28].

Observe that the cross-product of three vectors is antisymmetric and distributive over addition, that is, $\lfloor a b c\rfloor=$ $-\lfloor b a c\rfloor=\lfloor b c a\rfloor$, and $\left\lfloor a b\left(\alpha_{1} c+\alpha_{2} d\right)\right\rfloor=\alpha_{1}\lfloor a b c\rfloor+\alpha_{2}\lfloor a b d\rfloor$.

Definition 2 (Bracket) The bracket of four vectors $a, b, c$, and $d$ is defined as:

$$
[a b c d]=a \cdot\lfloor b c d\rfloor=\left|\begin{array}{llll}
a_{x} & a_{y} & a_{z} & a_{w} \\
b_{x} & b_{y} & b_{z} & b_{w} \\
c_{x} & c_{y} & c_{z} & c_{w} \\
d_{x} & d_{y} & d_{z} & d_{w}
\end{array}\right|
$$

According to this definition, four points, say $a, b, c$, and $d$, are coplanar if, and only if, $[a b c d]=0$. Then, we also have by duality that the four planes dual to points $a, b, c$ and $d$, intersect in a point if, and only if, $[a b c d]=0$.

Now, let the set of platform attachments be partitioned in three sets involving a couple of legs each: $\mathcal{A}=\{a, b, c, d\}$, $\mathcal{B}=\{e, f, g, h\}$ and $\mathcal{C}=\{i, j, k, l\}$.

Definition 3 (Stewart Bracket) The Stewart bracket $\langle p, q, r\rangle$, where $p \in \mathcal{A}, q \in \mathcal{B}$ and $r \in \mathcal{C}$, is defined as the bracket of the dual points to the four planes defined by the points in the sets $\mathcal{A} \backslash\{p\}, \mathcal{B} \backslash\{q\}, \mathcal{C} \backslash\{r\}$, and $\{p, q, r\}$. To avoid ambiguities, points will always be sorted in lexicographic order in all operations.

For example, according to the above definition,

$$
\langle d e i\rangle=[\lfloor a b c\rfloor\lfloor d e i\rfloor\lfloor f g h\rfloor\lfloor j k l\rfloor] .
$$

Observe that the total number of Stewart brackets for the partition of the set of attachments given by $\mathcal{A}, \mathcal{B}$ and $\mathcal{C}$ is 64 , and the total number of possible partitions is 15 .

\section{The Primal Form of the Pure Condition}

Theorem 1 (Primal Form of the Pure Condition) The determinant of the geometric Jacobian of a Stewart platform with the labelling of attachments shown in Fig. 1 can be expressed as:

$$
\begin{aligned}
& {[a b c d][e f g i][h j k l]-[a b c d][e f h i][g j k l]-[a b c d][e f g j][h i k l]} \\
& +[a b c d][e f h j][g i k l]-[a b c e][d f g h][i j k l]+[a b d e][c f g h][i j k l] \\
& -[a b d f][c e g h][i j k l]+[a b c f][\operatorname{degh}][i j k l]-[a b c e][d g h i][f j k l] \\
& +[a b d e][c g h i][f j k l]-[a b d f][c g h i][e j k l]+[a b c f][d g h i][e j k l] \\
& +[a b c e][d g h j][f i k l]-[a b d e][c g h j][f i k l]+[a b d f][c g h j][e i k l] \\
& -[a b c f][d g h j][e i k l]+[a b c g][d e f i][h j k l]-[a b c h][d e f i][g j k l] \\
& -[a b d g][c e f i][h j k l]+[a b d h][c e f i][g j k l]-[a b c g][d e f j][h i k l] \\
& +[a b c h][d e f j][g i k l]+[a b d g][c e f j][h i k l]-[a b d h][c e f j][g i k l] \\
& =0 \text {. }
\end{aligned}
$$

Proof: See [8].

As mentioned in the introduction, the primal form of the pure condition is unique up to syzygies. This means that there are different equivalent pure conditions that might vary in their number of terms. Up to our knowledge, the 16-term pure condition presented in [18] is the one with the lowest number of terms. The one given above has 24 terms but, though it is not the shortest one, it usually leads to the simplest results when applied to platforms with coincident attachments.

\section{The DuAl Form of the Pure Condition}

Lemma 1 The pure condition of a Stewart platform with topology

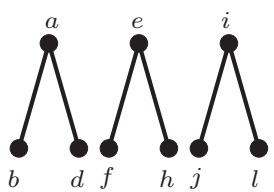

can expressed as $-[$ abde $][$ aehi $][$ fijl $]+[a b d f][$ aehi $][$ eijl $]+$ $[$ abde $][$ aefi $][$ hijl $]-[$ abdh $][$ aefi $][$ eijl $]=0$. This condition can be rewritten as $[\lfloor a b d\rfloor\lfloor a e i\rfloor\lfloor e f h\rfloor\lfloor i j l\rfloor]=0$ which will be called the pure condition in dual form for the above topology.

Proof: After some algebraic manipulations, this result follows from the analysis for this topology presented in [22].

Note that the condition in the above Lemma can be expressed, using Stewart brackets, simply as $\langle a e i\rangle=0$, after setting $c=a, g=e$, and $k=i$.

Lemma 2 The pure condition of a Stewart platform with topology

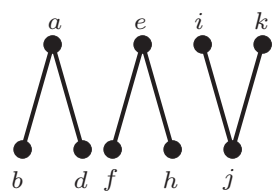

can expressed as $[a b d e][a e h j][f i j k]-[a b d f][a e h j][e i j k]-$ $[$ abde $][$ aef $j][$ hijk $]+[a b d h][$ aef $j][$ eijk $]=0$. This condition can be rewritten as $[\lfloor a b d\rfloor\lfloor a e j\rfloor\lfloor e f h\rfloor\lfloor i j k\rfloor]=0$ which will be called the pure condition in dual form of the above topology.

Proof: After some algebraic manipulations, this result follows from the analysis for this topology presented in [22]. 
Using Stewart brackets, the condition in the above Lemma can be simply expressed as $\langle a e j\rangle=0$, after setting $c=a$, $g=e$, and $l=j$.

Theorem 2 (Dual Form of the Pure Condition) The determinant of the geometric Jacobian of a Stewart platform with the labelling of attachments shown in Fig. 1 can be expressed as:

$$
\begin{aligned}
& -\langle a e i\rangle+\langle a e j\rangle+\langle a e k\rangle-\langle a e l\rangle+\langle a f i\rangle-\langle a f j\rangle \\
& -\langle a f k\rangle+\langle a f l\rangle+\langle a g i\rangle-\langle a g j\rangle-\langle a g k\rangle+\langle a g l\rangle \\
& -\langle a h i\rangle+\langle a h j\rangle+\langle a h k\rangle-\langle a h l\rangle+\langle b e i\rangle-\langle b e j\rangle \\
& -\langle b e k\rangle+\langle b e l\rangle-\langle b f i\rangle+\langle b f j\rangle+\langle b f k\rangle-\langle b f l\rangle \\
& -\langle b g i\rangle+\langle b g j\rangle+\langle b g k\rangle-\langle b g l\rangle+\langle b h i\rangle-\langle b h j\rangle \\
& -\langle b h k\rangle+\langle b h l\rangle+\langle c e i\rangle-\langle c e j\rangle-\langle c e k\rangle+\langle c e l\rangle \\
& -\langle c f i\rangle+\langle c f j\rangle+\langle c f k\rangle-\langle c f l\rangle-\langle c g i\rangle+\langle c g j\rangle \\
& +\langle c g k\rangle-\langle c g l\rangle+\langle c h i\rangle-\langle c h j\rangle-\langle c h k\rangle+\langle c h l\rangle \\
& -\langle d e i\rangle+\langle d e j\rangle+\langle d e k\rangle-\langle d e l\rangle+\langle d f i\rangle-\langle d f j\rangle \\
& -\langle d f k\rangle+\langle d f l\rangle+\langle d g i\rangle-\langle d g j\rangle-\langle d g k\rangle+\langle d g l\rangle \\
& -\langle d h i\rangle+\langle d h j\rangle+\langle d h k\rangle-\langle d h l\rangle=0
\end{aligned}
$$

Proof: The proof of this theorem can be graphically summarized as indicated in Fig. 2. Starting from the dual pure conditions for the topologies in the top row, the dual pure condition for the generic 6-6 Stewart platform is obtained.

Using Lemma 1 and 2, the dual pure conditions of the following two topologies
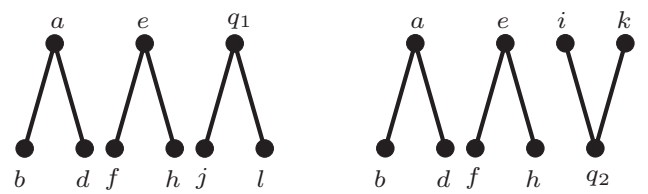

are

$$
\wp_{1}=\left[\lfloor a b d\rfloor\left\lfloor a e q_{1}\right\rfloor\lfloor e f h\rfloor\left\lfloor q_{1} j l\right\rfloor\right]
$$

and

$$
\wp_{2}=\left[\lfloor a b d\rfloor\left\lfloor a e q_{2}\right\rfloor\lfloor e f h\rfloor\left\lfloor i q_{2} k\right\rfloor\right],
$$

respectively.

Now, let $q_{1}$ and $q_{2}$ be collinear with $i$ and $k$, and with $j$ and $l$, respectively. That is,

$$
\begin{aligned}
& q_{1}=\alpha_{1} i+\alpha_{2} k \\
& q_{2}=\alpha_{3} j+\alpha_{4} l
\end{aligned}
$$

Then,

$$
\begin{array}{r}
\wp_{1}=\quad \alpha_{1}^{2}[\lfloor a b d\rfloor\lfloor\text { aei }\rfloor\lfloor e f h\rfloor\lfloor i j l\rfloor] \\
-\alpha_{1} \alpha_{2}[\lfloor a b d\rfloor\lfloor\text { aei }\rfloor\lfloor e f h\rfloor\lfloor j k l\rfloor] \\
\left.\left.+\alpha_{1} \alpha_{2}[\lfloor a b d\rfloor\lfloor\text { aek }\rfloor \text { ef } h\rfloor i j l\right\rfloor\right] \\
\left.\quad-\alpha_{2}^{2}[\lfloor a b d\rfloor\lfloor\text { aek }\rfloor\lfloor e f h\rfloor j k l\rfloor\right]
\end{array}
$$

and

$$
\begin{aligned}
\wp_{2}= & -\alpha_{3}^{2}[\lfloor\text { abd }\rfloor\lfloor a e j\rfloor\lfloor\text { ef } h\rfloor\lfloor i j k\rfloor] \\
& +\alpha_{3} \alpha_{4}[\lfloor\text { abd }\lfloor\lfloor a e j\rfloor\lfloor\text { ef } h\rfloor\lfloor i k l\rfloor] \\
& -\alpha_{3} \alpha_{4}[\lfloor\text { abd }\lfloor\text { ael }\rfloor \text { ef } h\rfloor\lfloor i j k\rfloor] \\
& +\alpha_{4}^{2}[\lfloor\text { abd }\lfloor\text { ael }\rfloor \text { ef } h\rfloor\lfloor k l\rfloor] .
\end{aligned}
$$

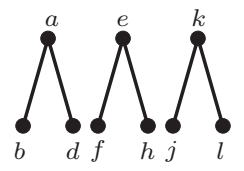
have that topologies

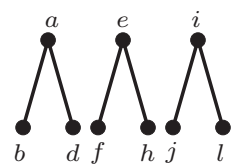

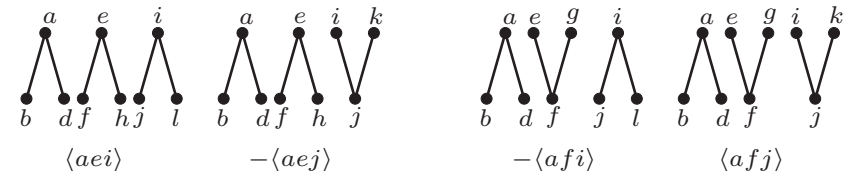
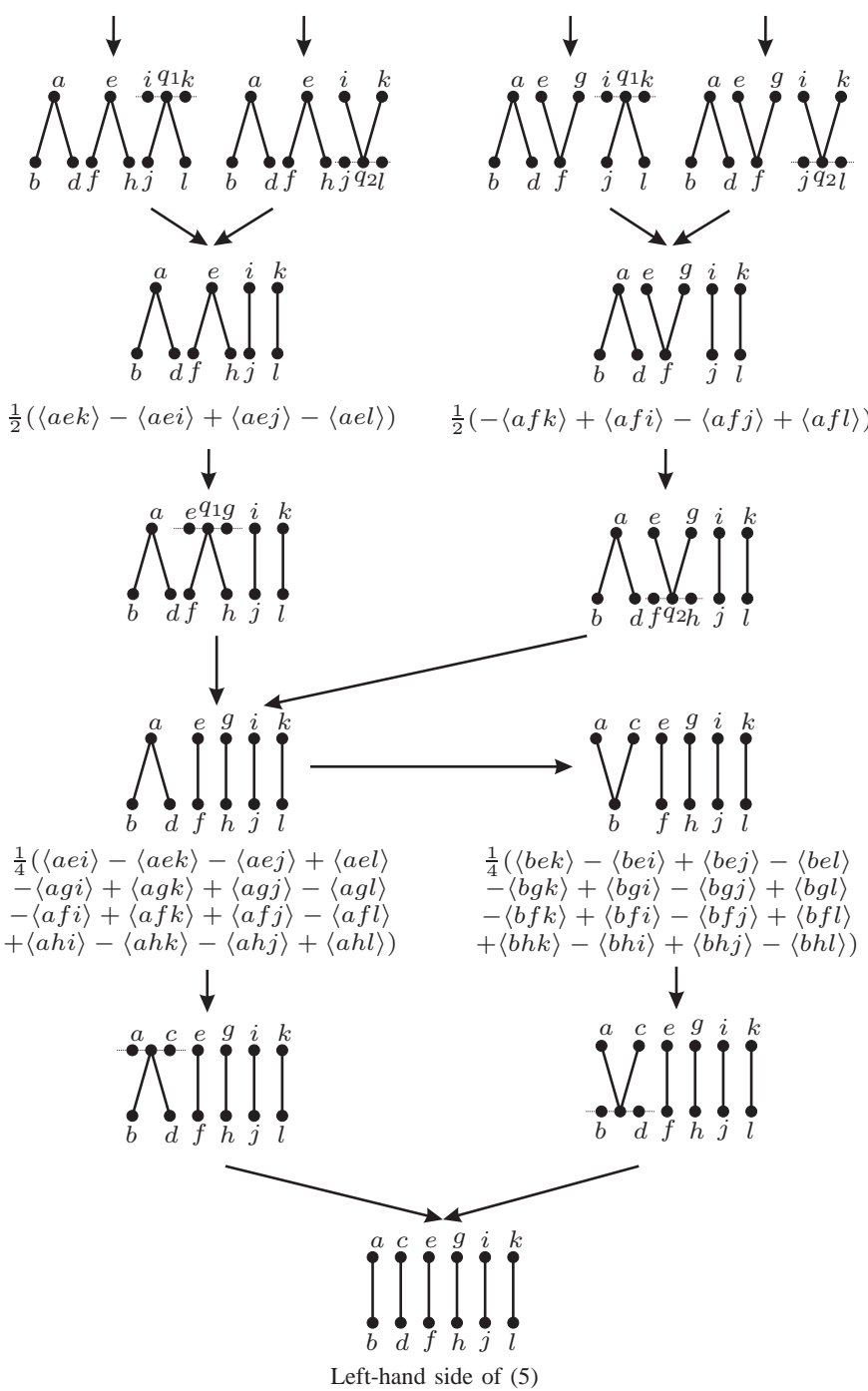

Fig. 2. Sequence of generalizations used to prove Theorem 2.

On the other hand, using the multilinearity property of the Jacobian determinants for the above two topologies, we also

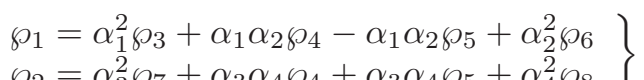

where $\wp_{3}, \ldots, \wp_{8}$ are the pure conditions of the following
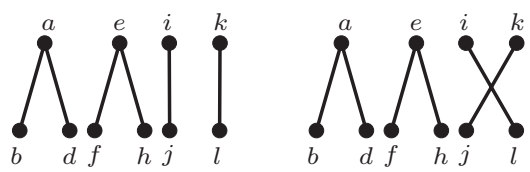

Preprint submitted to IEEE Transactions on Robotics. Received: April 13, 2012 11:07:05 PST 
respectively. Since, according to Lemma 1 and Lemma 2,

$$
\begin{aligned}
& \wp_{3}=[\lfloor a b d\rfloor\lfloor a e i\rfloor\lfloor\text { ef } h\rfloor\lfloor i j l\rfloor], \\
& \wp_{6}=-[\lfloor a b d\rfloor\lfloor a e k\rfloor\lfloor e f h\rfloor\lfloor j k l\rfloor], \\
& \wp_{7}=-[\lfloor a b d\rfloor\lfloor a e j\rfloor\lfloor e f h\rfloor\lfloor i j k\rfloor], \\
& \wp_{8}=[\lfloor a b d\rfloor\lfloor a e l\rfloor\lfloor e f h\rfloor\lfloor i k l\rfloor],
\end{aligned}
$$

then

$$
\begin{aligned}
\wp_{1} & \left.=\alpha_{1}^{2}[\lfloor a b d\rfloor\lfloor a e i\rfloor\lfloor e f h\rfloor \text { ijl }\rfloor\right] \\
& +\alpha_{1} \alpha_{2} \wp_{4} \\
& -\alpha_{1} \alpha_{2} \wp_{5} \\
& \quad-\alpha_{2}^{2}[\lfloor a b d\rfloor\lfloor a e k\rfloor\lfloor e f h\rfloor\lfloor j k l\rfloor]
\end{aligned}
$$

and

$$
\begin{aligned}
\wp_{2}= & -\alpha_{3}^{2}[\lfloor a b d\rfloor\lfloor a e j\rfloor\lfloor e f h\rfloor\lfloor i j k\rfloor] \\
& +\alpha_{3} \alpha_{4} \wp_{4} \\
& +\alpha_{3} \alpha_{4} \wp_{5} \\
& \left.+\alpha_{4}^{2}[\lfloor a b d\rfloor\lfloor\text { ael }\rfloor\lfloor e f h\rfloor i k l\rfloor\right] .
\end{aligned}
$$

Now, equating the right hand sides of (8) and (11), we get $[\lfloor a b d\rfloor\lfloor a e k\rfloor\lfloor e f h\rfloor\lfloor i j l\rfloor]-[\lfloor a b d\rfloor\lfloor a e i\rfloor\lfloor e f h\rfloor\lfloor j k l\rfloor]=\wp_{4}-\wp_{5}$, and equating the right hand sides of (9) and (12),

$[\lfloor a b d\rfloor\lfloor a e j\rfloor\lfloor e f h\rfloor\lfloor i k l\rfloor]-[\lfloor a b d\rfloor\lfloor a e l\rfloor\lfloor e f h\rfloor\lfloor i j k\rfloor]=\wp_{4}+\wp_{5}$.

Using Stewart brackets, after setting $c=a$, and $g=e$, the above two equations can be written as

$$
\left.\begin{array}{l}
\langle a e k\rangle-\langle a e i\rangle=\wp_{4}-\wp_{5} \\
\langle a e j\rangle-\langle a e l\rangle=\wp_{4}+\wp_{5}
\end{array}\right\}
$$

This linear system, when solved for $\wp_{4}$ and $\wp_{5}$, yields

$$
\begin{array}{r}
\wp_{4}=\frac{1}{2}(\langle a e k\rangle-\langle a e i\rangle+\langle a e j\rangle-\langle a e l\rangle) \\
\wp_{5}=\frac{1}{2}(-\langle a e k\rangle+\langle a e i\rangle+\langle a e j\rangle-\langle a e l\rangle)
\end{array}
$$

Proceeding in a similar way from the pure conditions in dual form of the topologies $(\triangle: \therefore)$ and ( $:: 2)$, it is obtained that of $(\$::: \AA$. Likewise, the pure conditions in dual form of

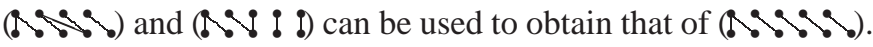
The obtained expression, by simply renaming labels, also leads to the dual pure condition of ( $\because \because 9)$. Then, the pure conditions for the last two topologies can be used to finally obtain the general dual pure condition in equation (5).

Corollary 1 The dual pure condition is the result of equating to zero the addition of all different Stewart brackets that can be formed for the partition of the set of attachments given by $\mathcal{A}, \mathcal{B}$ and $\mathcal{C}$. If $\mathcal{A}, \mathcal{B}$ and $\mathcal{C}$ are chosen so that they involve different couples of legs, a different but equivalent condition with the same number of terms is obtained. Thus, there are 15 equivalent pure conditions in dual form with the same number of terms.

Analogously to what happened to the pure condition in primal form, applying the dual pure condition to platforms where some of the attachments coincide leads to simplifications. In all cases, the appropriate pure condition must be chosen, from all possible equivalent pure conditions, to obtain the maximum simplification. The situation is quite complicated when dealing with the pure condition in primal form because this involves the use of syzygies. Nevertheless, when dealing with the pure condition in dual form this task is simplified because the number of equivalent conditions is 15 . As a general rule, it is advisable to minimize the number of legs sharing one attachment that appear in different partitions of the set of attachments.

\section{CHOICE OF PRIMAL VS. DUAL}

In Table I, a list of all possible topologies for the Stewart platform with the resulting pure conditions in primal and dual form is shown. Two main families of topologies can be drawn from this table:

1) A family of 21 topologies whose pure conditions, either in primal or in dual form, simplify to a single term. This family can be subdivided in three non-disjoint sets:

a) A set of 13 topologies whose primal pure condition simplify to a single term. For all members of this family the geometric conditions associated with their singularities can be expressed as the degeneracy of three tetrahedra. For example, a Stewart platform with topology

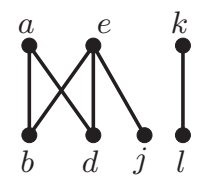

is in a singularity if, and only if, any of the three sets of points $\{a, b, d, e\},\{a, e, k, l\}$ or $\{b, d, e, j\}$ lie on a plane.

b) A set of 15 topologies whose dual pure condition simplify to a single term. Observe how this family includes the three 3-3 topologies and six of the seven 4-3 topologies. For all members of this family the geometric conditions associated with their singularities can be expressed as the degeneracy of a single tetrahedron. For example, a Stewart platform with topology

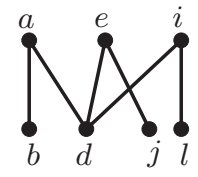

is in a singularity if, and only if, the tetrahedron with plane faces defined by the four sets of three points $\{a, b, d\},\{d, e, j\},\{d, i, l\}$ and $\{a, e, i\}$ has null volume (i.e.,the four planes are either coplanar, or intersect in a single line, or in a point).

c) A set of 7 topologies whose pure condition, both in pure and dual form, simplify to a single term. For example, a Stewart platform with topology

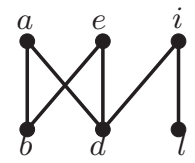


TABLE I

ALL STEWART PLATFORM TOPOLOGIES AND THEIR PURE CONDITIONS IN PRIMAL AND DUAL FORMS

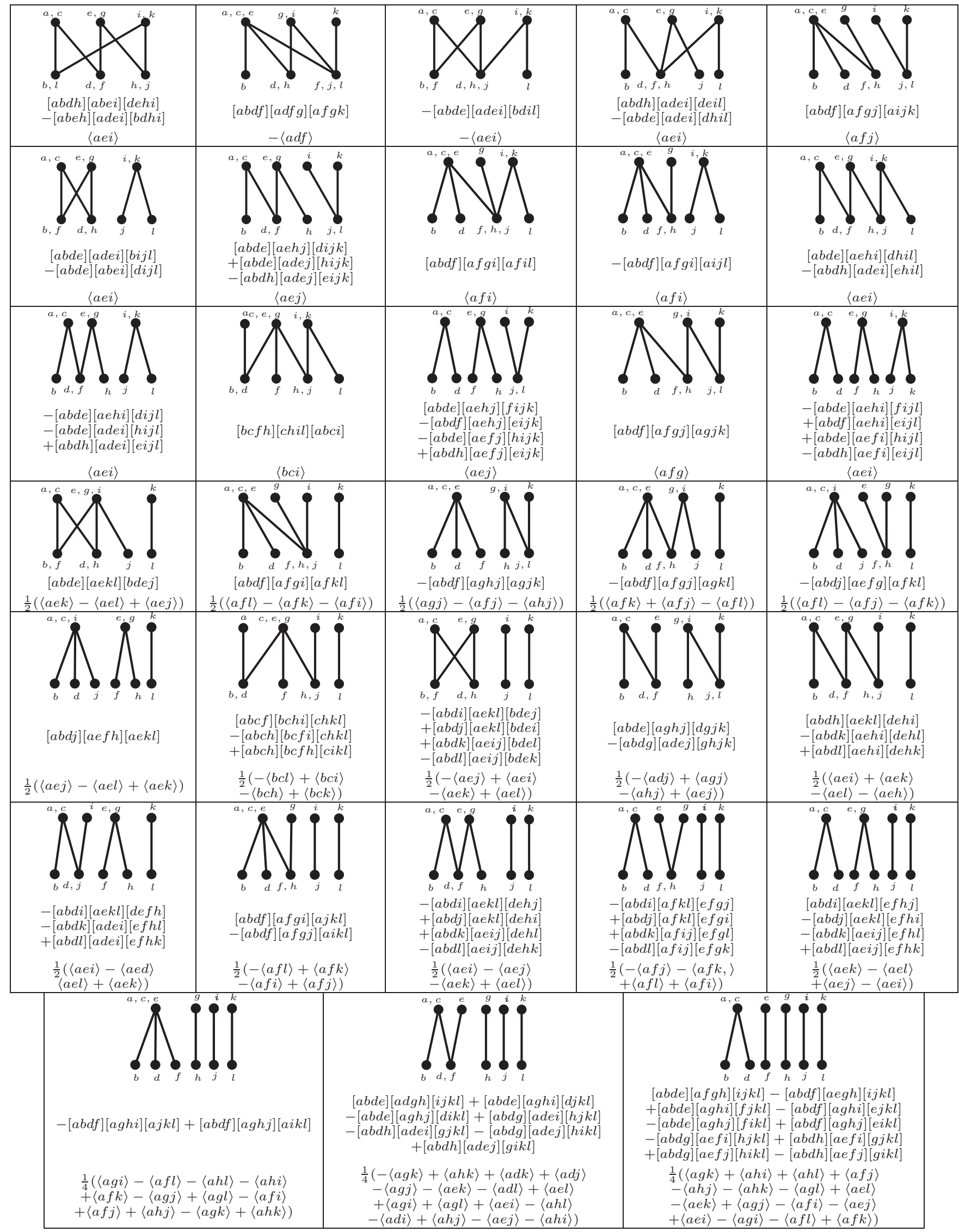

Preprint submitted to IEEE Transactions on Robotics. Received: April 13, 2012 11:07:05 PST 
is in a singularity if, and only if, any of the three sets of points in $\{a, b, d, e\},\{a, d, e, i\}$ or $\{b, d, i, l\}$ lie on a plane, or if, and only if, the tetrahedron with plane faces defined by the four sets of three points $\{a, b, d\},\{b, d, e\},\{d, i, l\}$ and $\{a, e, i\}$ has null volume.

2) A family of 12 topologies whose pure conditions, neither in primal nor in dual form, simplify. This family can be further subdivided into two sets:

a) A set of 9 topologies whose dual pure condition contains four terms. In all these cases, two planes are common to all four terms which simplifies the geometric interpretation of their singularities. This represents an important family of platforms which, thanks to the derived pure condition in dual form, can be treated in a unified way. The first example in the next section analyzes one of these topologies. The same analysis is valid for all other members of this set.

b) A set of three topologies (those in the last row in Table I) whose dual pure condition contains more than four terms. While the analysis, using the pure condition in its primal form, of these topologies seems to require a case-by-case treatment, the use of the dual form permits a unified analysis which becomes evident after analyzing the set of topologies. This is discussed at the end of the next section.

A particular set of seven different topologies, with common geometric interpretation for their singularities, was analyzed in [29] using Grassmann-Cayley algebra. It can be checked that this set is a subfamily of the 9 topologies whose pure condition in dual form has four terms and that we have just identified.

\section{EXAMPLES: SingUlarity ANALYSIS OF TWO 4-6 STEWART PLATFORMS}

Consider the Stewart platform in Fig. 3. It has the following topology:

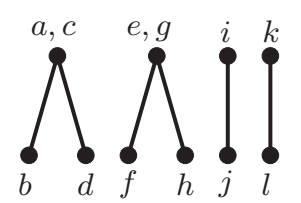

According to Table I, the primal pure condition for this topology can be expressed as:

$$
\begin{aligned}
& {[a b d i][a e k l][e f h j]-[a b d j][a e k l][e f h i]} \\
& \quad-[a b d k][a e i j][e f h l]+[a b d l][a e i j][e f h k]=0
\end{aligned}
$$

This topology was analyzed in [29] where it was shown that (13), after non-trivial manipulations using syzygies, reduces to:

$$
[a e i j][p q k l]-[a e k l][p q i j]=0
$$

where $p$ and $q$ are points on the line given by the intersection of the planes $a b d$ and $e f h$.

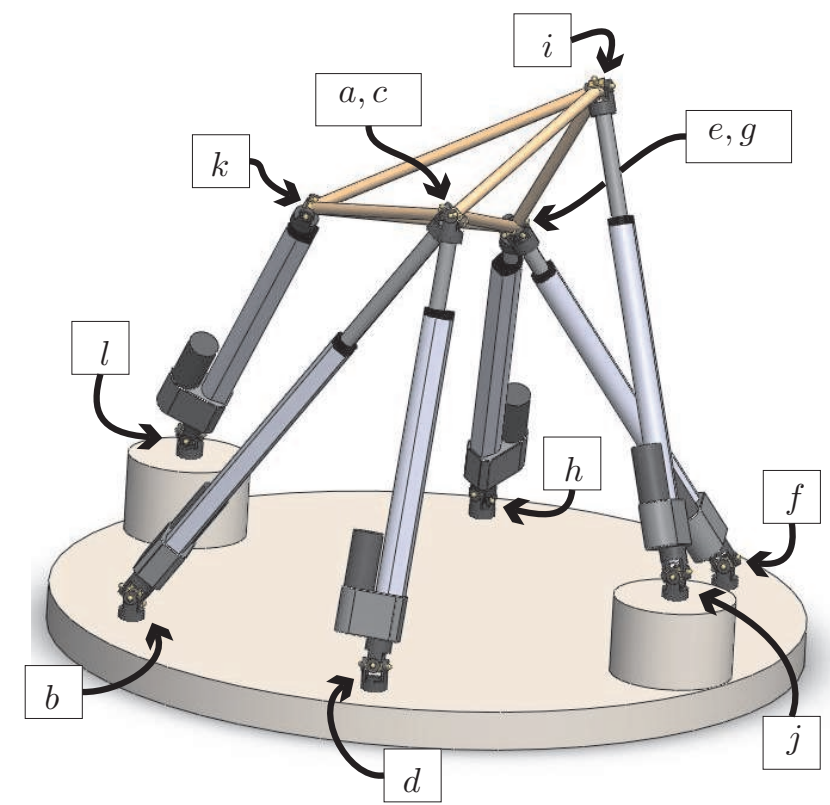

\begin{tabular}{|c||c|c|c|}
\hline$l$ & 0 & -4 & 1 \\
\hline$b$ & $2 \sqrt{3}$ & -2 & 0 \\
\hline$d$ & $2 \sqrt{3}$ & 2 & 0 \\
\hline$j$ & 0 & 4 & 1 \\
\hline$f$ & $-2 \sqrt{3}$ & 2 & 0 \\
\hline$h$ & $-2 \sqrt{3}$ & -2 & 0 \\
\hline
\end{tabular}

\begin{tabular}{|c||c|c|c|}
\hline$a^{\prime}, c^{\prime}$ & 2 & 0 & 0 \\
\hline$e^{\prime}, g^{\prime}$ & -2 & 0 & 0 \\
\hline$i^{\prime}$ & 0 & 2 & 1 \\
\hline$k^{\prime}$ & 0 & -2 & 1 \\
\hline
\end{tabular}

Fig. 3. A 4-6 Stewart platform and coordinates of the base and platform attachments in their local reference frames. The two coincident attachments on the moving platform can be implemented using double gimbals as explained in [30].

This topology was also studied in [31] where the singularity condition was expressed as the addition of four terms, each of them being the product of two $3 \times 3$ determinants, which can be rewritten in terms of dot and cross products of several vectors related with the geometry of the robot and then with the eigenvectors of a certain matrix.

Now, as an alternative to the above formulations, we can directly use the pure condition in dual form. According to Table I, it can be expressed as:

$$
\begin{aligned}
& (1 / 2)([\lfloor a b d\rfloor\lfloor a e k\rfloor\lfloor e f h\rfloor \text { ijl }\rfloor]+[\lfloor a b d\rfloor\lfloor a e j\rfloor\lfloor e f h\rfloor\lfloor i k l\rfloor] \\
- & {[\lfloor a b d\rfloor\lfloor a e i\rfloor\lfloor e f h\rfloor\lfloor j k l\rfloor]-[\lfloor a b d\rfloor\lfloor a e l\rfloor\lfloor e f h\rfloor\lfloor i j k\rfloor])=0 }
\end{aligned}
$$

which can be rewritten as

$$
p_{1} \cdot\left(\left\lfloor p_{2} p_{3} p_{4}\right\rfloor+\left\lfloor p_{5} p_{3} p_{6}\right\rfloor-\left\lfloor p_{7} p_{3} p_{8}\right\rfloor-\left\lfloor p_{9} p_{3} p_{10}\right\rfloor\right)=0,
$$

or, equivalently, as

$$
p_{3} \cdot\left(\left\lfloor p_{1} p_{2} p_{4}\right\rfloor+\left\lfloor p_{1} p_{5} p_{6}\right\rfloor-\left\lfloor p_{1} p_{7} p_{8}\right\rfloor-\left\lfloor p_{1} p_{9} p_{10}\right\rfloor\right)=0
$$

where

$$
\begin{aligned}
& p_{1}=\lfloor a b d\rfloor, \quad p_{2}=\lfloor a e k\rfloor, \quad p_{3}=\lfloor e f h\rfloor, \quad p_{4}=\lfloor i j l\rfloor, \\
& p_{5}=\lfloor a e j\rfloor, \quad p_{6}=\lfloor i k l\rfloor, \quad p_{7}=\lfloor a e i\rfloor, \quad p_{8}=\lfloor j k l\rfloor, \\
& p_{9}=\lfloor\text { ael }\rfloor, \quad p_{10}=\lfloor i j k\rfloor .
\end{aligned}
$$


Since the sum of point/plane coordinates always correspond to the coordinates of a point/plane, both

$$
r_{1}=\left\lfloor p_{2} p_{3} p_{4}\right\rfloor+\left\lfloor p_{5} p_{3} p_{6}\right\rfloor-\left\lfloor p_{7} p_{3} p_{8}\right\rfloor-\left\lfloor p_{9} p_{3} p_{10}\right\rfloor
$$

and

$$
r_{2}=\left\lfloor p_{1} p_{2} p_{4}\right\rfloor+\left\lfloor p_{1} p_{5} p_{6}\right\rfloor-\left\lfloor p_{1} p_{7} p_{8}\right\rfloor-\left\lfloor p_{1} p_{9} p_{10}\right\rfloor
$$

also represent two points/planes. Then, the analyzed platform will be in a singularity if point $r_{1}$ lies on the plane defined by $p_{1}$ or, equivalently, if point $r_{2}$ lies on plane $p_{3}$. Then, the singularity condition can be simply expressed as $r_{1} \cdot p_{1}=0$ or, equivalently, as $r_{2} \cdot p_{3}=0$. Moreover, observe that by construction $r_{1} \cdot p_{3}=0$ and $r_{2} \cdot p_{1}=0$. Then, it can be concluded that the analyzed robot is in a singularity if, and only if, both $r_{1}$ and $r_{2}$ lie simultaneously on $p_{1}$ and $p_{3}$.

Note that if any of the planes defined in (15) is degenerate, it gives a null-vector, but equation (14) is still well-defined. If $p_{1}$ or $p_{3}$ are null vectors (i.e., if $a, b$, and $d$, or $d, f$, and $h$ are aligned) the manipulator will be in a singularity. The degeneracy of any other plane in (15) does not imply that the condition (14) is satisfied.

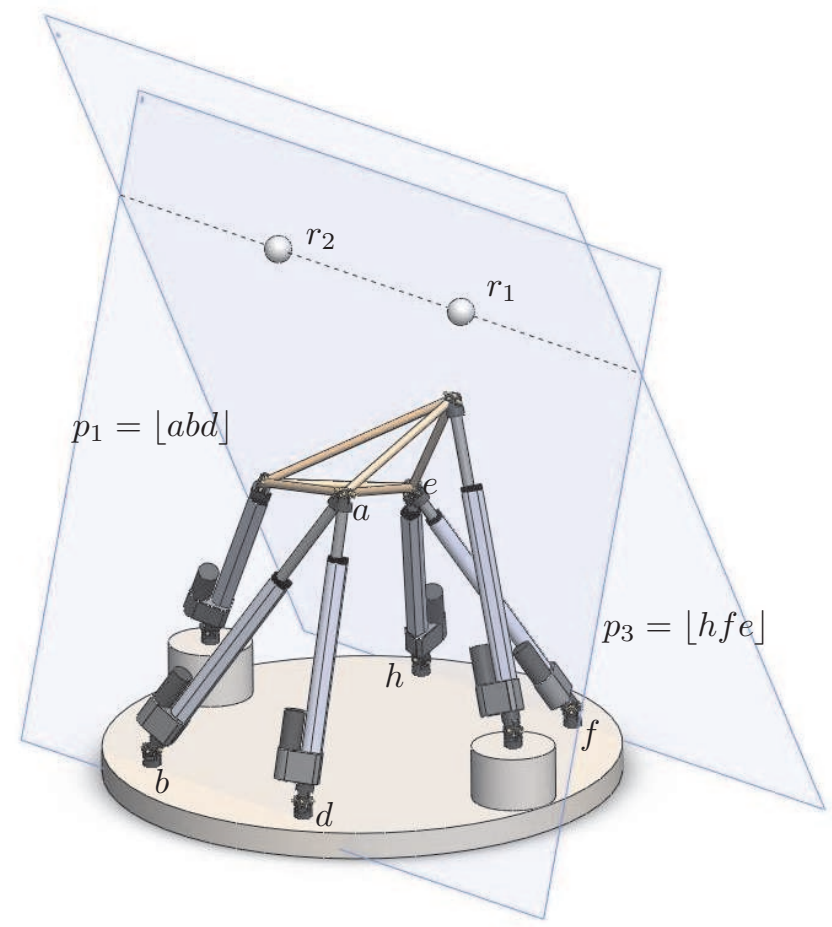

Fig. 4. The analyzed 4-6 Stewart platform in a singular configuration. In this case, $p_{x}=0, p_{y}=0.792$, and $p_{z}=5$.

To get some practical insight, let us suppose that the coordinates of the attachments for the analyzed robot, in their local reference frames, are the ones in Fig. 3. Let us also suppose that the orientation of the moving platform with respect to be base is fixed to be $\operatorname{Rot}\left(z, \frac{\pi}{6}\right) \operatorname{Rot}\left(x, \frac{\pi}{6}\right)$. In other words, according to the notation used in Fig. $3, a^{T}=$ $\operatorname{Trans}\left(p_{x}, p_{y}, p_{z}\right) \operatorname{Rot}\left(z, \frac{\pi}{6}\right) \operatorname{Rot}\left(x, \frac{\pi}{6}\right)\left(a^{\prime}\right)^{T}$ and, similarly, for the other platform attachments. Then, the moving platform is only allowed to translate and

$$
p_{1}=\left(\begin{array}{c}
-2-4 p_{z} \\
0 \\
4\left(p_{x}-\sqrt{3}\right) \\
4 \sqrt{3}\left(1+2 p_{z}\right)
\end{array}\right) \text {, }
$$

$$
\begin{gathered}
p_{3}=\left(\begin{array}{c}
-2+4 p_{z} \\
0 \\
-4\left(p_{x}+\sqrt{3}\right) \\
4 \sqrt{3}\left(2 p_{z}-1\right)
\end{array}\right), \\
r_{1}=\left(\begin{array}{c}
16\left(p_{x}-3.46 p_{z}+3.46\right)\left(7.07 p_{x}+6.92 p_{x} p_{z}-\right. \\
\left.1.73 p_{z}+17.73-4 p_{x} p_{y}-3 p_{y}\right) \\
-384 p_{z}^{2} p_{y}+221.71 p_{y}^{2} p_{z}-203.42 p_{z}^{2}+ \\
110.85 p_{x} p_{z} p_{y}+331.16 p_{y} p_{z}-64 p_{x} p_{y}^{2}- \\
221.71 p_{y}^{2}-1525.1 p_{z}-604.71 p_{x} p_{z}+ \\
276.84 p_{y}+144.57 p_{x} p_{y}+1050.4 p_{x}+ \\
126.85 p_{x}^{2}+1728.6 \\
32\left(-1+2 p_{z}\right) \\
\left(3.46 p_{y} p_{z}-3.46 p_{y}-6 p_{z}^{2}-5.32 p_{z}+\right. \\
\left.18.32-p_{x} p_{y}+1.73 p_{x} p_{z}+3.26 p_{x}\right) \\
-219.71 p_{z}^{2}+62.85 p_{y} p_{z}-32.573 p_{x} p_{z}+ \\
141.16 p_{z}-18.143 p_{x} p_{y}-62.85 p_{y}+ \\
302.56+248 . p_{x}+27.714 p_{x}^{2}
\end{array}\right),
\end{gathered}
$$

and

$$
r_{2}=\left(\begin{array}{c}
-16\left(p_{x}+3.46 p_{z}\right)\left(7.07 p_{x}+6.92 p_{x} p_{z}\right. \\
\left.-1.73 p_{z}+17.73-4 p_{x} p_{y}-3 p_{y}\right) \\
-384 p_{z}^{2} p_{y}+221.71 p_{y}^{2} p_{z}+683.42 p_{z}^{2}- \\
110.85 p_{x} p_{z} p_{y}-564.84 p_{y} p_{z}+64 . p_{x} p_{y}^{2}- \\
444.0 p_{z}+383.00 p_{x} p_{z}+300.56 p_{y}- \\
336.57 p_{x} p_{y}+302.15 p_{x}-588.0+1.15 p_{x}^{2} \\
-16\left(1+2 p_{z}\right) \\
\left(-6.92 p_{y} p_{z}+3.92 p_{y}+12 p_{z}^{2}+8.91 p_{z}-\right. \\
\left.18.91-2 p_{x} p_{y}+3.46 p_{x} p_{z}+0.53 p_{x}\right) \\
-164.29 p_{z}^{2}+158.85 p_{y} p_{z}-78.28 p_{x} p_{z}- \\
42.27 p_{z}+82.143 p_{x} p_{y}-62.85 p_{y}+ \\
302.56-265.15 p_{x}-27.714 p_{x}^{2}
\end{array}\right) .
$$

Now, it can be checked, using a computer algebra system, that $\operatorname{det}(\mathbf{J})=\frac{1}{2} r_{1} \cdot p_{1}=\frac{1}{2} r_{2} \cdot p_{3}$ where the rows of $\mathbf{J}$ are the Plücker coordinates of the leg lines.

As explained above, $r_{1}$ and $r_{2}$ lie, by construction, on planes $p_{3}$ and $p_{1}$, respectively, but if $r_{1}$ also lies on $p_{3}$ or, equivalently, $r_{2}$ on $p_{1}$, the robot is in a singularity. This is the situation depicted in Fig. 4.

The above analysis can be extended to the 9 topologies whose dual pure condition contains four terms because, in all these cases, two planes are common to all four terms.

Now, consider the Stewart platform in Fig. 5. It has the following topology:

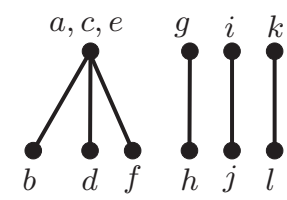

Here, we have chosen this topology, over the other two in the same set of topologies identified in the previous section, 
because it corresponds to the decoupled Stewart platform. Indeed, three prismatic actuators control the location of point where three attachments coincide, which can be seen as the center of the robot, and the other three control the orientation of the moving platform. Thus, if the first three actuators are blocked, we can regard the result as a parallel spherical robot where two pyramid-like rigid bodies are connected together by a spherical pair at the common apex.

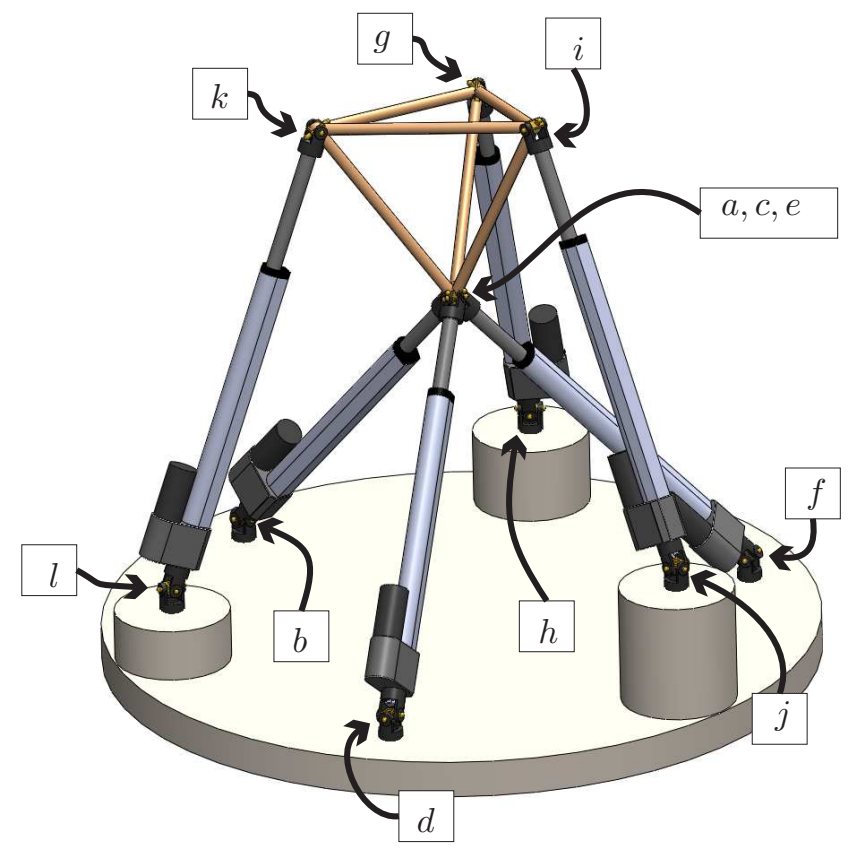

Fig. 5. A decoupled Stewart platform.

The forward kinematics of this robot was first solved by Innocenti and Parenti-Castelli [32], and Wohlhart [33]. Alici and Shirinzadeh presented a method based on the determinant of the Jacobian to plot the singularity locus in terms of the moving platform orientation using Euler angles [34]. Later, Ben-Horin and Shoham found a compact singularity condition for this robot using Grassmann-Cayley algebra [35].

According to Table I, the dual pure condition for this topology can be expressed as:

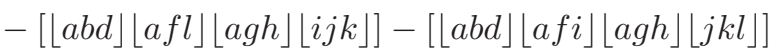

$$
\begin{aligned}
& +[\lfloor a b d\rfloor\lfloor a f h\rfloor\lfloor a g i\rfloor\lfloor j k l\rfloor]+[\lfloor a b d\rfloor\lfloor a f k\rfloor\lfloor a g h\rfloor\lfloor i j l\rfloor] \\
& -[\lfloor a b d\rfloor\lfloor a f h\rfloor\lfloor a g k\rfloor\lfloor i j l\rfloor]+[\lfloor a b d\rfloor\lfloor a f g\rfloor\lfloor a h j\rfloor\lfloor i k l\rfloor] \\
& +[\lfloor a b d\rfloor\lfloor a f g\rfloor\lfloor a h k\rfloor\lfloor i j l\rfloor]-[\lfloor a b d\rfloor\lfloor a f g\rfloor\lfloor a h l\rfloor\lfloor i j k\rfloor] \\
& +[\lfloor a b d\rfloor\lfloor a f h\rfloor\lfloor a g l\rfloor\lfloor i j k\rfloor]-[\lfloor a b d\rfloor\lfloor a f h\rfloor\lfloor a g j\rfloor\lfloor k l\rfloor] \\
& +[\lfloor a b d\rfloor\lfloor a f j\rfloor\lfloor a g h\rfloor\lfloor i k l\rfloor]-[\lfloor a b d\rfloor\lfloor a f g\rfloor\lfloor a h i\rfloor\lfloor j k l\rfloor]=0
\end{aligned}
$$

which can be rewritten as:

$$
\begin{aligned}
p_{1} \cdot & \left(-\left\lfloor p_{2} p_{3} p_{4}\right\rfloor-\left\lfloor p_{5} p_{3} p_{6}\right\rfloor+\left\lfloor p_{7} p_{8} p_{6}\right\rfloor\right. \\
& +\left\lfloor p_{9} p_{3} p_{10}\right\rfloor-\left\lfloor p_{7} p_{11} p_{10}\right\rfloor+\left\lfloor p_{12} p_{13} p_{14}\right\rfloor \\
& \quad+\left\lfloor p_{12} p_{15} p_{10}\right\rfloor-\left\lfloor p_{12} p_{16} p_{4}\right\rfloor+\left\lfloor p_{7} p_{17} p_{4}\right\rfloor \\
\quad & \left.-\left\lfloor p_{7} p_{18} p_{14}\right\rfloor+\left\lfloor p_{19} p_{3} p_{14}\right\rfloor-\left\lfloor p_{12} p_{20} p_{6}\right\rfloor\right)=0
\end{aligned}
$$

where

$$
\begin{aligned}
& p_{1}=\lfloor a b d\rfloor, \quad p_{2}=\lfloor a f l\rfloor, \quad p_{3}=\lfloor a g h\rfloor, \quad p_{4}=\lfloor i j k\rfloor, \\
& p_{5}=\lfloor\text { afi }\rfloor, \quad p_{6}=\lfloor j k l\rfloor, \quad p_{7}=\lfloor\text { afh }\rfloor, \quad p_{8}=\lfloor\text { agi }\rfloor, \\
& p_{9}=\lfloor a f k\rfloor, \quad p_{10}=\lfloor i j l\rfloor, \quad p_{11}=\lfloor\text { agk }\rfloor, \quad p_{12}=\lfloor a f g\rfloor, \\
& p_{13}=\lfloor a h j\rfloor, \quad p_{14}=\lfloor i k l\rfloor, \quad p_{15}=\lfloor a h k\rfloor, \quad p_{16}=\lfloor a h l\rfloor, \\
& p_{17}=\lfloor a g l\rfloor, \quad p_{18}=\lfloor a g j\rfloor, \quad p_{19}=\lfloor\text { afj }\rfloor, \quad p_{20}=\lfloor\text { ahi }\rfloor \text {. }
\end{aligned}
$$

Then, although this dual pure condition have more than four terms, they all have one plane as a common factor. Thus, the singularities of this robot can also be geometrically interpreted as the incidence of a point with a plane. This point is the result of dualizing a plane resulting from adding 12 planes defined by sets of three attachments, instead of only four planes as in the previous example.

Alternatively, according to Table I, the primal pure condition for this topology can be expressed as:

$$
\begin{aligned}
& -[a b d f][a g h i][a j k l]+[a b d f][a g h j][a i k l] \\
& \quad=[a b d f]([a g h j][a i k l]-[a g h i][a j k l])=0
\end{aligned}
$$

This condition is more compact and easier to interpret geometrically than (16) because it factors into two terms that can be analized independently. Observe that the condition $[a b d f]=0$ corresponds to the singularities of the translational part of the robot. The other term can be simplified using Grassman-Cayley algebra (see [35] for details) but, using the concepts introduced in this paper as an alternative, we can rewrite it as:

$$
\begin{aligned}
& -[j a g h][i a k l]+[\text { iagh }][j a k l] \\
& =(-j \cdot\lfloor\text { agh }\rfloor)(i \cdot\lfloor\text { akl }\rfloor)+(i \cdot\lfloor\text { agh }\rfloor)(j \cdot\lfloor\text { akl }\rfloor)=0 .
\end{aligned}
$$

Since $(u \times v) \cdot(w \times s)=(u \cdot w)(v \cdot s)-(u \cdot s)(v \cdot w)$, then this singularity condition can be finally expressed as:

$$
(i \times j) \cdot(\lfloor a g h\rfloor \times\lfloor a k l\rfloor)=0 .
$$

This condition constitutes a new convenient alternative to the one derived in [35] as it can be readily interpreted geometrically using no other tools than standard vector algebra. Then, although the singularity analysis of the topologies in the last row of Table I can be unified using the dual pure condition, its case-by-case treatment based on the primal pure condition still seems advantageous mainly when it is combined with the concepts introduced in this paper.

\section{CONCLUSION}

We have presented a new singularity condition for the generic 6-6 Stewart platform as a linear combination of $4 \times 4$ determinants involving plane coordinates, which has been called pure condition in dual form. This expression has been proved to be of interest to obtain geometric interpretations of the singularity conditions.

Finally, it is worth realizing that the pure condition, either in its primal or dual forms, can be applied to any parallel manipulator with line-based singularities, a type of manipulators first characterized in [36]. This fact has been used in [9] to analyze lower mobility platforms with three legs, first by using screw algebra to obtain the governing lines, and then applying the pure condition in its primal form to the result. The interest of 
using the dual form of the pure condition instead is certainly a point that deserves further attention. In our opinion, this might lead to a generalization of the results presented in [37].

\section{REFERENCES}

[1] J.-P. Merlet, Parallel Robots, Springer, 2000.

[2] J.-P. Merlet and C.M. Gosselin, "Parallel mechanisms and robots," Chap. 12 in Springer Handbook of Robotics, B. Siciliano and O. Khatib (Eds.), Springer, 2008.

[3] R. Ranganath, P.S. Nair, T.S. Mruthyunjaya, and A. Ghosal, "A forcetorque sensor based on a Stewart platform in a near-singular configuration," Mechanism Machine Theory, Vol. 39, No. 9, pp. 971-998, 2004.

[4] J.-P. Merlet, "Jacobian, manipulability, condition number, and accuracy of parallel robots," ASME J. of Mechanical Design, vol. 128, no. 1, pp. 199-206, 2006.

[5] J.C. Faugère and D. Lazard, "The combinatorial classes of parallel manipulators," Mechanism Machine Theory, vol. 30, no. 6, pp. 765-776, 1995.

[6] P. Bosscher and I. Ebert-Uphoff, "A novel mechanism for implementing multiple collocated spherical joints," Proc. of the IEEE Int. Conf. on Robotics and Automation, pp. 336-341, 2003.

[7] J. Borràs, Singularity-Invariant Leg Rearrangements in Stewart-Gough Platforms, PhD Thesis, Technical University of Catalonia, to appear, 2011.

[8] P. Ben-Horin and M. Shoham, "Singularity condition of six degreeof-freedom three-legged parallel robots based on Grassmann-Cayley algebra," IEEE Trans. on Robotics, vol. 22, no. 4, pp. 577-590, 2006.

[9] D. Kanaan, P. Wenger, S. Caro, and D. Chablat, "Singularity analysis of lower-mobility parallel manipulators using Grassmann-Cayley algebra," IEEE Transactions on Robotics, vol. 25, no. 5, pp. 995-1004, 2009.

[10] B. St-Onge Mayer and C.M. Gosselin,"Singularity analysis and representation of the general Gough-Stewart platform," International Journal of Robotics Research, vol. 19, no. 3, pp. 271-288, 2000.

[11] H. Li, C.M. Gosselin, M.J. Richard, and B. St-Onge Mayer, "Analytic form of the six-dimensional singularity locus of the general GoughStewart platform," ASME Journal of Mechanical Design, vol. 128, no. 1, pp. 279-287, 2006.

[12] H. Pottmann and J. Wallner, Computational Line Geometry, Springer Verlag, Berlin, 2001.

[13] A. Dandurand, "The rigidity of compound spatial grids," Structural Topology, vol. 10, pp. 41-56, 1984.

[14] C. Gibson and K. H. Hunt, "Geometry of screw systems-I, screws: Genesis and geometry," Mechanism and Machine Theory, vol. 25, no. 1, pp. 1-10, 1990.

[15] C. Gibson and K. Hunt, "Geometry of screw systems-II, screws: classification of screw systems," Mechanism and Machine Theory, vol. 25, no. 1 , pp. 11-27, 1990.

[16] J.-P. Merlet, "Singular configurations of parallel manipulators and Grassmann geometry," International Journal of Robotics Research, vol. 8, no. 5 , pp. 45-56, 1989.

[17] J-P. Merlet, "On the infinitesimal motion of a parallel manipulator in singular configurations," Proc. of the IEEE Int. Conf. on Robotics and Automation, Nice, pp. 320-325, 1992.

[18] D. Downing, A. Samuel, and K. Hunt, "Identification of the special configurations of the octahedral manipulator using the pure condition," International Journal of Robotics Research, vol. 21, no. 2, pp. 147-159, 2002.

[19] E. Staffetti and F. Thomas, "Kinestatic analysis of serial and parallel robot manipulators using grassmann-cayley algebra," in International Symposium on Advanced Robot Kinematics, 2000, pp. 17-26.

[20] N. White, "The bracket of 2-extensors," Congressus Numerantium, vol. 40, pp. 419-428, 1983.

[21] F. Thomas, "An approach to the movers' problem that combines Oriented Matroid Theory and Algebraic Geometry," Proc. of the 1995 IEEE Int. Conf. on Robotics and Automation, Vol. III, pp. 2285-2293, 1995.

[22] P. Ben-Horin and M. Shoham, "Singularity analysis of a class of parallel robots based on Grassmann-Cayley algebra," Mechanism and Machine Theory, vol. 41, no. 8, pp. 958-970, 2006.

[23] R. Di Gregorio, "Singularity-locus expression of a class of parallel mechanisms," Robotica, vol. 20, pp. 323-328, 2002

[24] J. Borràs, F. Thomas, and C. Torras, "Straightening-free algorithm for the singularity analysis of Stewart-Gough platforms with collinear/coplanar attachments," in International Workshop on Computational Kinematics, 2009.
[25] J. Semple and G. Kneebone, Algebraic Projective Geometry, Oxford University Press, 1952.

[26] H.S.M. Coxeter, Introduction to Geometry, John Wiley, 1961.

[27] M.Z. Williams and F.M. Stein, "A triple product of vectors in fourspace," Mathematics Magazine, vol. 37, no. 4, pp. 230-235, 1964.

[28] M. Johnson, "Proof by duality or the discovery of 'new' theorems," Mathematics Today (Bulletin of the Institute of Mathematics and its Applications), vol. 32, no. 11, pp. 171 - 174, 1996.

[29] P. Ben-Horin and M. Shoham, "Application of Grassmann-Cayley algebra to geometrical interpretation of parallel robot singularities," International Journal of Robotics Research, vol. 28, no. 1, pp. 127$141,2009$.

[30] K.H. Hunt and P.R. McAree, "The octahedral manipulator: geometry and mobility," The International Journal of Robotics Research, vol. 17, no. 8, pp. 868-885, 1998 .

[31] R. Di Gregorio, "Singularity locus of 6-4 fully-parallel manipulators," in International Symposium on Advanced Robot Kinematics, 2010, pp. 437-445.

[32] C. Innocenti and V. Parenti-Castelli, "Echelon form solution of direct kinematics for the general fully-parallel spherical wrist," Mechanism and Machine Theory, vol. 28, no. 4, pp. 553-561, 1993.

[33] K. Wohlhart, "Displacement analysis of the general spherical Stewart platform," Mechanism and Machine Theory, vol. 29, no. 4, pp. 581589, 1994.

[34] G. Alici and B. Shirinzadeh, "Loci of singular configurations of a 3-DOF spherical parallel manipulator," Robotics and Autonomous Systems, vol. 48, pp. 77-91, 2004.

[35] P. Ben-Horin and M. Shoham, "Singularity of a class of Gough-Stewart platforms with three concurrent joints," Advances in Robot Kinematics, Lenarcic, J. and Roth, B. (eds), Ljubljana, Slovenia, pp. 265-274, 2006.

[36] F. Hao and J. McCarthy, "Conditions for line-based singularities in spatial platform manipulators," Journal of Robotic Systems, vol. 15, no. 1, pp. 43-55, 1998.

[37] I. Ebert-Uphoff, J.-K. Lee, and L. H. Lipkin, "Characteristic tetrahedron of wrench singularities for parallel manipulators with three legs," Proceedings of the Institution of Mechanical Engineers C, Journal of Mechanical Engineering Science, vol. 126, no. C1, pp. 81-93, 2002. 